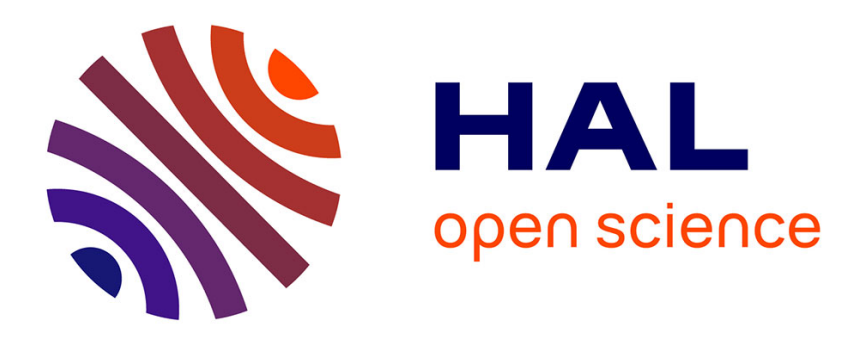

\title{
Intimité, mise en scène et distance dans la relation politique au Liban.
}

\author{
Isabelle Rivoal
}

\section{To cite this version:}

Isabelle Rivoal. Intimité, mise en scène et distance dans la relation politique au Liban.. Leaders et partisans au Liban, Karthala-IFPO-IISMM, pp.139-165, 2012. halshs-00769845

\section{HAL Id: halshs-00769845 \\ https://shs.hal.science/halshs-00769845}

Submitted on 3 Jan 2013

HAL is a multi-disciplinary open access archive for the deposit and dissemination of scientific research documents, whether they are published or not. The documents may come from teaching and research institutions in France or abroad, or from public or private research centers.
L'archive ouverte pluridisciplinaire HAL, est destinée au dépôt et à la diffusion de documents scientifiques de niveau recherche, publiés ou non, émanant des établissements d'enseignement et de recherche français ou étrangers, des laboratoires publics ou privés. 


\title{
Intimité, mise en scène et distance dans la relation politique au Liban*
}

Isabelle Rivoal

\author{
"Walid Joumblatt, il est comme le soleil : \\ quand on est trop loin, on gèle, quand on est trop près, on brûle... "
}

\section{Approches du patronage politique et de la domination au Liban}

L'analyse du politique au Liban s'est essentiellement construite sur l'étude des relations de patronage et des formes de domination qui l'organisent. Plus précisément, elle s'est attachée à comprendre les ambiguïtés des rapports entre les leaders politiques, ces za'îm susceptibles d'être élus à la Chambre des députés ${ }^{1}$, et leurs hommes de main, les qabadây; relation prenant sens dans le contrôle exercé sur des masses de partisans. Si cette relation a été privilégiée comme manifestation du pouvoir c'est bien en raison de la singularité du Liban, que l'on peut qualifier de société non hobbesienne, dans le sens où l'État et la société civile n'y sont pas distingués ${ }^{2}$. L'État au Liban n'est en effet pas autre chose qu'un système de patronage ${ }^{3}$ et cette caractéristique en fait certainement une "République précaire ", selon la formule célèbre de Michael Hudson ${ }^{4}$. Autrement dit, la permanence d'un fait communautaire, reflété dans les bases légales de l'État, entravant l'émergence d'une centralité politique (partant, d'une société civile), permettrait d'expliquer la prédominance du patronage comme lieu $d u$ politique par excellence. La plupart des sociologies politiques du Liban ont ainsi entrepris de rendre compte de l'articulation entre réalités locales (l'échelle des sociétés partisanes, localisées ${ }^{5}$ ) et réalités nationales (les institutions gouvernementales à Beyrouth) en termes de contrôle social fondé sur la violence et de modalités de médiation comme pivot nécessaire au système confessionnel. La politique, domaine de l'action contingente cette fois pour reprendre la

\footnotetext{
* Je voudrais remercier Laurence Caillet, Raymond Jamous et Christine Jungen pour leur lecture attentive et leurs suggestions critiques sur les premières versions de cet article.

${ }^{1}$ Selon la qualification donnée par Peter Gubster (1973).

2 Pour une discussion du principe de société civile antérieur au libéralisme, voir Donegani et Sadoun, 2007, p. 167-168.

${ }^{3}$ Selon la thèse de Michael Gilsenan (1977).

${ }^{4}$ Hudson, 1968.

${ }^{5}$ Par « localités » j'entends ici tous les regroupements politiques concrets construits sur la relation complexe et orientée entre un leader politique et ceux qui le soutiennent plus ou moins visiblement. Ces formes peuvent se modeler sur une communauté religieuse, une région, un parti, sans jamais en être une expression exclusive. C'est à ce titre qu'on les considérera comme des expressions politiques localisées.
} 
distinction de Julien Freud ${ }^{6}$, n'est jamais au Liban que l'expression d'un rapport de force qui se stabilise dans une formule de gouvernement (la fameuse sîgha lubnâniyya) de manière toujours provisoire.

Qu'est-ce qui lie un leader politique à ceux qui se reconnaissent comme ses partisans ? Comment se construisent et se perpétuent les loyautés partisanes ? Quelles sont les manifestations, dans la vie quotidienne, d'une relation politique caractérisée par sa dimension personnelle ? Comment surtout rendre compte de cette dimension personnelle dans une organisation politique réputée fondée sur la coercition et la violence ? On doit sûrement à Michael Gilsenan l'une des tentatives les plus abouties pour(de) saisir les fondements de la domination comme pratique sociale au Liban et non seulement(uniquement) comme dimension inhérente aux relations de patronage. C'est sur le NordLiban, avant le déclenchement de la guerre civile et dans un milieu organisé autour de la grande propriété terrienne, que son enquête se concentre. Les hiérarchies sociales qu'il observe sont encore entièrement indexées sur le contrôle des ressources agricoles avec les seigneurs (bey) ayant autorité sur leur domaine, les hommes de main (agha) qui sont en charge d'administrer et de surveiller ce domaine pour le compte du seigneur et enfin les paysans (fallahîn) qui l'exploitent. Dans ce contexte, l'autorité et la légitimité sont fortement hiérarchisées. La domination est surtout expérimentée comme un état de fait (thingness), une essence donnée indépendamment de tout acte de volition : " les bey et les agha sont ce qu'ils sont ». Elle est présentée comme étant dans la nature des choses, et cette qualité précède l'interprétation que les gens en donnent ${ }^{7}$. Cela ne veut pas dire qu'elle puisse pour autant demeurer dans l'implicite. Elle doit au contraire être montrée, exposée, performée pour être ce qu'elle est et, par la narration de coups d'éclat des dominants, devenir la matrice de l'histoire locale, voire son fondement idéologique ${ }^{8}$. La domination est ainsi constamment reproduite et rejouée dans les situations sociales les plus diverses et dans ses formes les plus arbitraires, selon une esthétique de la violence qui confère au seigneur une dimension d'icône ${ }^{9}$.

Tout comme Michael Johnson dans son étude classique des relations patron-client dans le quartier sunnite de Musaytba à Beyrouth ${ }^{10}, \mathrm{c}^{\prime}$ est à partir de cette position de I'homme de main, du qabadây assurant le relais entre le patron politique et sa clientèle par la médiation et le contrôle adossé au recours à la violence, que $\mathrm{M}$. Gilsenan donne à voir l'ambivalence des formes de domination dans un Liban rural. Sa lecture des relations de patronage met en lumière une double extériorité du point de vue local : l'extériorité concrète des za'îm (les bey) dont la domination est fondée, à l'origine, sur l'octroi d'une fonction militaire ou fiscale par le pouvoir ottoman, et qui doivent résider à Beyrouth, où se joue la compétition politique dont dépend leur statut ; l'extériorité symbolique des paysans qui demeurent hors de l'histoire locale - sans profondeur généalogique - et hors des narrations. Dans le Akkar, les qabadây (agha), dont le statut est entièrement fondé sur la domination comme discours et pratique de la violence, sont en charge d'articuler et de perpétuer "l'ordre du monde ". Ce sont finalement ces hommes de main qui apparaissent comme les acteurs pivot de la configuration locale

\footnotetext{
${ }^{6}$ Freund, 1965, p. 45.

7 Michael Gilsenan prend soin ici de spécifier que son usage des notions d'autorité et de légitimité n'a pas pour vocation de saisir la vie sociale autrement que comme construction de sens partagée (Gilsenan, 1986).

${ }^{8}$ Même, les actes sont perpétrés dans le seul but de devenir des histoires, comme autant de repères constitutifs d'un « nous » local inscrit dans la permanence par la répétition (Gilsenan, 1989).

${ }^{9}$ Gilsenan, 1996, p. 3-22, et 1986.

10 Johnson, 1986.
} 
des relations de patronage. Ceux aussi qui ont le plus à perdre dans un système où le pouvoir personnel n'est sanctifié que par le code de l'honneur, la mise en scène agonistique de la masculinité et de l'individualisme.

Les transformations économiques profondes ${ }^{11}$ qui ont quasiment réduit à néant l'assise politique conférée par la propriété terrienne à vocation agricole et les années de guerre civile ont certainement modifié la figure du qabadây, transformant de ce fait la relation entre le za îm et ses partisans, notamment dans les régions rurales. L'expansion et le développement des réseaux clientélistes libanais a connu un coup d'arrêt en 1975 en raison de l'effondrement de l'État, vers lequel est(était) orienté tout le système des relations politiques. Plus exactement, l'État comme forme institutionnelle est demeuré, mais il n'était plus le lieu privilégié de l'expression des rapports de force, qui se mesuraient désormais dans le cadre des affrontements miliciens ${ }^{12}$. La conséquence la plus notable de cet effondrement a été le resserrement des différents réseaux partisans à un niveau plus localisé ${ }^{13}$. Les leaders politiques sont tous plus ou moins devenus des notables urbains, figures de médiateurs au service d'une économie de commerce et de finance. Les hommes de main doivent désormais avoir des compétences d'administrateurs. Quant à "l'esthétique de l'honneur ", elle s'est "démocratisée " avec la migration rurale ${ }^{14}$. Dès lors, la dimension communautaire semble être l'élément qui permet le mieux d'expliquer comment les patrons politiques ont été en mesure de maintenir leur légitimité en dépit de la nature inéquitable et coercitive de leur domination ${ }^{15}$. Ce qui revient à poser la question de la compréhension des loyautés politiques et de leur construction congruente en "sens communautaire ". II ne peut s'agir du produit unique de la domination, tout comme il est difficile de penser que ces leaders ne représentent plus les sociétés locales qui pérennisent leur prédominance ${ }^{16}$.

Le pouvoir politique est plus qu'une affaire de contrôle et de représentation au Liban. Poussée à la limite de la caricature, la logique de cette lecture assimilant la relation de patronage à une modalité de pouvoir coercitif conduit à dénoncer le «verrouillage par les élites politiques de l'ordre

\footnotetext{
${ }^{11}$ Pour une histoire économique de cette transformation, voir Dib, 2004, p. 91 sq.

${ }^{12}$ On a ainsi débattu pour savoir si les miliciens étaient ou non des figures équivalentes à l'homme de main traditionnel dans l'espace urbain (Beyhum, 1999). Et plus généralement, on a cherché à savoir si les violences de la guerre civile étaient une forme exacerbée des modalités de domination et de coercition déjà présentes ou une forme radicalement nouvelle reflétant les transformations de la société (Picard, 2001). Mais dans ce débat encore, c'est le point d'articulation social où se manifestent avec le plus d'éclat les formes du contrôle - et en l'occurrence, le passage d'un contrôle des hommes à un contrôle de l'espace - qui monopolise l'analyse. La relation entre la société et son leadership n'est pas comprise autrement que comme une relation de pouvoir de nature coercitive.

${ }^{13}$ Ces phénomènes ont été particulièrement bien documentés dans les régions chrétiennes sous le contrôle des Forces libanaises et dans la région du Chouf sous le contrôle de la milice du Parti socialiste progressiste de Walid Joumblatt. Voir notamment Harik, 1993, et El-Ghoussayni, 1988.

${ }^{14}$ Selon la thèse de Michael Johnson, qui analyse l'émigration rurale(l'exode rural) comme le changement majeur ayant modifié l'ordre social au Liban. Tandis qu'à la Montagne le seigneur et ses hommes de main sont les seuls à revendiquer publiquement l'honneur et la masculinité, à Beyrouth, la multiplication des patrons politiques " urbanisés " a amené chaque homme à jouer l'honneur pour lui-même et à l'exprimer violemment au nom de la pureté de la communauté à laquelle il appartient (Johnson, 2001, p. 40-47).

${ }^{15}$ Khalaf, 2001, notamment p. 42-45 ; Kingston, 2001 ; Johnson, 2001.

${ }^{16}$ Selon la thèse d'Élizabeth Picard, pour qui « les masses sont assujetties à un cadre communautaire " qui a pour fonction d'empêcher leur accès à la scène politique en légitimant la prédominance des pôles du pouvoir, élites traditionnelles et miliciennes confondues (Picard, 1997).
} 
communautaire " afin "d'empêcher l'accès du peuple à la sphère politique " ${ }^{17}$. À vouloir retrouver le schéma hobbesien au Liban, on ne peut faire autrement que de s'enfermer dans le constat de l'absence d'une société civile; celle-ci n'aurait en effet d'expression possible qu'en dehors de la logique des communautés. Cette expression existe bien, mais elle demeure un fait politique résiduel, qui ne permet pas de rendre compte des formes de l'accord social ${ }^{18}$ dans le contexte libanais, ni de comprendre la pérennité des relations de patronage. Le pouvoir doit être saisi comme un principe d'organisation sociale, comme un lieu d'élaborations idéologiques et mémorielles, voire comme un idéal ou une esthétique. II organise tout autant la vie politique que la vie quotidienne des gens parce que n'importe quelle manifestation ordinaire de l'existence partagée y est d'emblée pourvue de caractère politique. C'est ce " caractère politique " particulier qu'il convient de cerner. Compris à ce niveau, le leader n'est pas seulement un médiateur entre l'échelle locale, communautaire, et l'échelle nationale, il n'est pas uniquement le représentant d'une 'asabiyya ou de loyautés traditionnelles. Son autorité agit comme une référence et cette référence est construite dans la relation qui « attache » le leader et ses partisans ${ }^{19}$; elle n'est jamais extérieure ou lointaine ni ne s'impose unilatéralement, contre une société qui ne saurait être autonome qu'en devenant civile.

\section{1-Le leader dans le quotidien des partisans : les formes d'une relation intime}

Walid Joumblatt est l'un de ces hommes politiques que les Libanais désignent comme des "pôles " (aqtâb) parce qu'ils polarisent une clientèle à dominante régionale et communautaire. Son leadership reflète de manière assez typique la mutation d'une domination jadis fondée sur la propriété terrienne vers la constitution d'une société politique locale. II la reflète d'autant plus que cette lignée beylicale ${ }^{20}$ peut se targuer d'une indéniable longévité : les Joumblatt se sont installés dans le Chouf au début du XVII ${ }^{e}$ siècle à la faveur d'une alliance avec l'émir Fakhr al-Dîn II al-Ma'nî ${ }^{21}$ et ont émergé comme l'une des familles dominantes dans la région après les affrontements de $1711^{22}$. La trajectoire de la maison Joumblatt dans sa capacité à adapter les modalités de son leadership à la nouvelle donne politico-économique du Liban indépendant est bien connue: distribution des terres familiales par Kamal Joumblatt au nom d'un idéal socialiste ; création d'une structure partisane en 1949, le Parti socialiste progressiste qui deviendra pendant la guerre civile le cadre à l'intérieur duquel sera développée la milice et qui servira de base à l'établissement d'une véritable administration territoriale de 1983 à 1990; investissements économiques diversifiés

\footnotetext{
${ }^{17}$ Picard, 1994.

${ }^{18}$ Entendu ici dans le sens que lui a donné la sociologie pragmatique et qui suppose de concevoir l'espace social comme étant traversé par une multitude de disputes en situation pour instaurer des accords locaux toujours provisoires car indexés sur ces situations et les lectures divergentes de ce qui justifie l'action.

19 Selon la belle formule de Gérard Lenclud, "S'attacher. Le régime traditionnel de protection en Corse » (1993).

${ }^{20}$ Selon(D'après) le titre de bey (prononcé bek et signifiant chef de clan) donné par l'administration ottomane. Walid Joumblatt est ainsi appelé Walid bek par les gens de la Montagne.

${ }^{21}$ Hichi, 1973-1974, p. 56 sq.

${ }^{22}$ Lors de l'abolition du système muqâta'ajî en 1841, le clan Joumblatt contrôlait 200 villages et 30000 personnes (Khuri, 2004, p. 140).
} 
pendant et après la guerre, et contrôle des flux financiers octroyés au ministère des Déplacés et de la Réconciliation ${ }^{23}$.

Walid Joumblatt a donc tout, en apparence, du patron politique libanais installé à Beyrouth mais dépendant d'une clientèle électorale régionale. Héritier d'une "maison politique " historique au Liban, il s'est efforcé - non sans réussite - de jouer sur la scène nationale un rôle dépassant largement sa base clientéliste "naturelle ", en dépit de l'érosion de la structure partisane fondée par son père. Qu'on lui reproche son rôle de chef de guerre durant les années d'affrontement, qu'on raille ses renversements d'alliance politique, qu'on fustige son verbe ou son attitude souvent provocatrice, Walid Joumblatt a su s'imposer comme une "constante " dans la vie politique libanaise. On imagine qu'il lui serait aisé d'incarner cette figure de seigneur lointain, absorbé par les arcanes de la politique nationale et confiant à la structure partisane qu'il contrôle ou à ses hommes de main la charge d'assurer la fidélité de sa clientèle locale. Si la relation de patronage s'épuisait en effet dans l'échange comptable de services et de recommandations que la littérature décrit le plus souvent, si elle ne reposait que sur l'exercice de la coercition, le leader politique cesserait d'être une référence pertinente dès lors que la nécessité de la relation disparaîtrait.

C'est ce que j'ai longtemps supposé, jusqu'à ce que je décide, à la suite de plusieurs longs séjours dans la Montagne libanaise, d'aller enquêter à Sydney auprès de migrants originaires de la communauté druze libanaise. Sortis de "l'orbite politique locale " qui fait de Walid Joumblatt une référence politique centrale dans les discours des personnes que j'avais côtoyées dans le Chouf, ces migrants avaient un positionnement politique hors des contraintes de la relation de patronage et du système confessionnel, que je voulais observer par souci comparatif : que disait-on du patron dans un contexte où il n'est plus nécessaire comme garant et médiateur? J'ai ainsi rencontré Nabil lors d'une réunion à l'Association des druzes de Sydney. Quelque temps après avoir fait sa connaissance, cet homme bien établi, ayant réussi à fonder une petite entreprise locale, me convie à partager un déjeuner dominical avec sa famille. Nabil habite depuis plus de trente ans à Manly, une banlieue plutôt chic au nord de Sydney, loin des quartiers ouest de la ville où se concentrent les Libanais. L'ancienneté de sa migration, sa réussite, sa volonté de rompre géographiquement avec la communauté des Libanais de Sydney dessinent une trajectoire d'autonomisation et de distanciation presque paradigmatique. Pourtant, dès que mon hôte me fait entrer chez lui et m'invite à prendre place dans le salon pour y boire les rafraîchissements d'usage, j'ai l'impression de franchir une frontière physique, de quitter la rumeur de Sydney et d'être soudain arrivée dans le Chouf. J'observe. L'effet de familiarité ne tient pas au mobilier relativement dépouillé de la pièce. II ne tient pas tellement aux effluves qui me parviennent de la cuisine, mélange de café et de fritures pour le mezze. Non, il tient essentiellement aux photographies encadrées sur les murs et posées sur les commodes. Sur le mur principal, un cadre des religieux druzes les plus vénérés jouxte un portrait de Kamal Joumblatt. Et puis dans un cadre sur lequel mon hôte attire l'attention, ce portrait de la famille au grand complet entourant Walid Joumblatt. La photographie a été prise lors de leur dernière visite au Liban deux ans auparavant. Ce cliché, on le retrouve sur une commode de la cuisine, au milieu des

\footnotetext{
${ }^{23}$ Le ministère des Déplacés est créé le 7 juillet 1992 par décret du Conseil des ministres qui lui associe attribue un fonds pour financer le retour des déplacés et la reconstruction. Walid Joumblatt est nommé ministre des Déplacés, fonction qu'il occupera jusqu'en 1998 (Kanafani-Zahar, 2004).
} 
portraits scolaires des enfants. L'iconographie construit soudain une familiarité que je n'avais pas mesurée jusqu'alors.

Dans le cadre de son étude sur l'expérience migratoire, Ghassan Hage a enquêté sur la dimension de se sentir "chez-soi ", qu'il désigne comme "home-building", distinct du simple fait de construire une maison, " house-building ». Un " chez-soi » est avant tout une construction affective qui suppose de (re)construire un espace où l'on peut être en sécurité - dans le sens de ne pas avoir à y subir partout la loi de l'autre -, où l'on peut déployer une connaissance familière de l'espace, mais aussi où il est possible de faire régulièrement l'expérience d'un sens de la communauté (c'est-à-dire partager avec d'autres un ensemble de symboles, de références, une morale et des valeurs qui assoient une possibilité de communication maximale dans un quotidien désormais vécu ailleurs). En somme, se sentir " chez-soi » est une expérience qui relève de l'intime et des stratégies de redéploiement de ce qui en relève : distanciation, appropriation et possibilité d'un entre-soi ${ }^{24}$. Tout autant que le "ahlân wa sahlân " m'accueillant sur le seuil de la maison ou la saveur du taboulé qui allait m'être offert ensuite, mon sentiment d'être transportée au Liban lors de cette visite chez Nabil a été déclenché par la " présence " familière des cheikhs druzes, de Kamal bey et de Walid bey dans mon champ visuel immédiat. C'est parce que le "seuil » se trouvait marqué ici d'une manière plus évidente que j'ai réalisé avec acuité combien j'avais intériorisé une ${ }^{25}$ version d'être au Liban, qui passait par la présence des Joumblatt dans l'espace intime du "chez-soi ». Au cours de mes visites dans le Chouf, je m'étais habituée à voir ces portraits sur les murs - ou leur absence tout aussi ostentatoire comme les signes convenus de l'allégeance au leader régional - ou de la défiance à son encontre ; en somme, une affaire politique relevant du patronage.

La mise en scène la plus courante consiste à placer un portrait en noir et blanc de Kamal Joumblatt et à l'entourer de clichés du chef de famille et de son père défunt, dans une disposition triangulaire où le leader domine visuellement les autres portraits. Un portrait de Kamal Joumblatt indique le soutien à la maison Joumblatt. Un portrait de Walid Joumblatt indique une maison de partisans plus impliqués dans leur allégeance au leader politique. Avec le temps, on apprend à lire l'iconographie des salons libanais comme des points de repère dans une cartographie partisane. Les portraits de religieux sont-ils plus nombreux ou plus visibles que les portraits politiques ? Walid Joumblatt est-il photographié seul, avec son père, en compagnie du maître de maison ? Le portrait est-il une copie circulant largement ou un cliché plus privé ? Etc.

Les salons ont une fonction d'exposition en tant que pièce de réception. L'hôte se met en scène devant ses invités et indique qui il est et quelles sont ses allégeances. On pourrait imaginer que les portraits des leaders y soient cantonnés à ce titre, mais ce n'est pas le cas. Chez Nabil à Sydney, Walid Joumblatt est au centre de l'espace quotidien de manière redondante : sur la photo, il campe au milieu de la famille, et ce portrait se trouve comme démultiplié, entouré qu'il est par d'autres photos de famille, dans un ensemble disposé à côté de la table de la cuisine, lieu de la vie quotidienne. Ici, il ne s'agit plus de signaler une allégeance au visiteur ou de se plier à un affichage convenu comme on pourrait le supposer dans une maison du Chouf, par exemple. Dans un contexte de migration, il se révèle pleinement comme élément important du dispositif de construction de

\footnotetext{
${ }^{24}$ Hage, 1997.

25 "Une » version car chaque région libanaise a sa propre manière de constituer " son » Liban intime, comme chaque communauté libanaise raconte sa propre histoire du Liban ou se bat pour sa version idéale du Liban.
} 
l'intime, de ce qui est à soi, pour soi. Plus encore, cette disposition indique que la famille a besoin d'un tiers pour se constituer et être offerte aux regards. Le leader est plus que l'un des termes d'une relation qui définirait son client en tant que client : il est constitutif de la famille.

C'est en ceci que la référence aux hommes politiques ne relève pas exclusivement, au Liban, de l'espace masculin par excellence que sont les salons ${ }^{26}$. On les voit souvent dans les cuisines, petits portraits parfois fixés sur les frigos en compagnie de clichés de stars de la chanson ou d'acteurs de cinéma, ou dans des endroits plus inattendus encore où ils semblent avoir pour fonction de " tenir compagnie ". Dans le Chouf, je rendis un jour visite à une femme de Baaqline avec qui j'étais suffisamment familière pour qu'elle $m^{\prime}$ 'invitât à poursuivre avec elle la conversation dans le réduit où elle faisait ses fritures. Au-dessus de la grande bassine bouillonnante était épinglée une coupure de journal représentant Taymour, le fils aîné de Walid Joumblatt. Interrompant soudain l'anecdote qu'elle était en train de me raconter, elle arrêta son regard sur le portrait et tout en entreprenant de passer dessus le torchon qu'elle tenait à la main me dit avec tendresse : « oh, regarde ce que je fais au pauvre homme, il est tout couvert d'huile ! ".

Ces portraits ne sont pas seulement un élément du décor, ils en sont parfois un agent actif dans le sens où ils permettent d'inscrire au quotidien l'interaction avec le leader ; une forme de substitut qui rend possible justement l'actualisation de cette dimension familière et affectueuse. II semblait ainsi évident, dans la manière dont mon hôtesse a passé le chiffon en mouvements descendants, à droite et à gauche, qu'elle n'essuyait pas une coupure de journal mais bien un visage, dans une attitude pleine de tendresse. De tels gestes soulignent à quel point les personnages politiques sont plus qu'une autorité ; ils vivent avec les gens. La scène n'était pas sans évoquer la manière dont les vieilles femmes se comportent à l'égard de Walid Joumblatt, lorsqu'elles le croisent à l'occasion d'un meeting dans le village ou lui rendent visite à Moukhtara. Elles le font se pencher en avant afin de pouvoir lui embrasser le front et lui souhaiter longue vie. Mais à la différence du rapport à l'image dans un cadre privé, l'expression d'affection manifestée dans la rencontre réelle ne s'effectue pas sans marque de déférence voire de soumission à l'autorité : "Que Dieu allonge ta vie, et qu'il te garde au dessus de nos têtes, Walid bek ". Mais il n'y a pas que dans la relation symbolique mère-fils que l'affection et la proximité s'expriment. Je me souviens ainsi de ce matin où je sirotais mon café dans la cuisine de la famille qui m'héberge lors de mes séjours dans le Chouf. Marwan, le jeune fils de la famille, venait d'entrer à l'université et il s'était rapproché de l'organisation des jeunesses du PSP. II en avait rapporté un calendrier qui était affiché au beau milieu du mur faisant face au canapé. Lorsqu'il nous avait rejoints encore tout ensommeillé, et après nous avoir salués, il s'était exclamé en regardant le portrait du leader druze à la page de février : " bonjour ya Walid bey, en voilà une belle journée qui commence!" Dans ces deux scènes, l'image n'agit pas comme une référence pour l'extérieur, pour dire où l'on est et à quelle distance dans la chaîne des relations personnelles qui tissent)le rapport politique. Dans cet espace familier et féminin de la cuisine et de ses dépendances, les portraits affichés sans cadres ni décorum sont des supports permettant l'expression d'une

\footnotetext{
${ }^{26}$ C'est en ceci que la place accordée aux leaders politiques est spécifique au Liban. Chez les druzes d'Israël, les seules photographies de personnages politiques qui sont exposées dans les salons de réception, et toujours de manière discrète et isolée par rapport aux photos de famille, sont celles de Sultan al-Attrach et Kamal Joumblatt, références « historiques » pour les druzes de Syrie et du Liban. Leur présence serait incongrue dans les cuisines.
} 
dimension affective dans la relation au leader, que je n'ai jamais vu manifester avec les portraits bien encadrés dans les salons.

La construction de cette dimension intime ne passe pas uniquement par l'image occasionnellement transformée en substitut du leader auquel on s'adresse avec familiarité. Sa " parole » est également présente, de multiples façons, dans le quotidien. On ne peut pas dire d'Ibtissam qu'elle soit politiquement proche des Joumblatt ; son mari vient d'une famille qui compte deux frères ayant fait fortune dans l'activité bancaire et qui ayant) subi la violence de Walid Joumblatt pendant la guerre parce qu'ils refusaient de participer au financement de la milice ${ }^{27}$. Elle $\mathrm{m}^{\prime}$ explique ainsi qu'elle ne peut pas envoyer ses enfants dans les jeunesses du PSP, ou monter au palais de Moukhtara pour demander quoi que ce soit au bey. Mais elle est originaire d'une famille joumblatti-e et, me dit-elle, " Walid bey sait que je l'aime et il connaît ma position; je suis sûre qu'il comprend pourquoi je ne vais pas à Moukhtara, que je pense d'abord à l'avenir de mes enfants ${ }^{28}$ ". Comme les temps sont difficiles et qu'elle a des inquiétudes pour l'avenir, c'est auprès de l'enseignement de Kamal Joumblatt qu'elle se ressource cependant. "Tous les matins, je me lève à $6 \mathrm{~h}$ et je commence ma journée en lisant un texte du mu'allim ${ }^{29}$, et puis je fais du yoga. Après, je me sens plus calme pour affronter la journée. Sinon, je serais trop nerveuse. Kamal bey nous a montré la voie. " Ce mode de positionnement est certainement particulier à la figure de Kamal Joumblatt qui a su se construire une stature empruntant à la symbolique religieuse, amplifiée par une mort violente qui l'a transformé en martyr. Le représentant politique d'une communauté est ici transformé en guide spirituel dont les textes lus et relus, médités et incorporés par la pratique du yoga - d'une manière qui rappelle l'injonction à devenir le Livre de la sagesse faite aux religieux druzes - donnent un sens aux difficultés du quotidien.

Si la parole de Kamal Joumblatt, disponible par l'écrit, prédispose à ce traitement particulier dans la vie quotidienne, d'autres vecteurs peuvent également se prêter à une sorte de tête-à-tête intime avec le chef politique. On peut en rendre compte en observant le traitement des enregistrements des discours des hommes politiques qui circulent particulièrement chez les jeunes ${ }^{30}$. Mixée, mise en musique et vendue dans les échoppes sous forme de CD, la parole de Walid Joumblatt ou de ses ministres est réappropriée par les jeunes partisans au terme de ce travail de montage : les passages préférés sont extraits des discours et deviennent des hits. Si Marwan écoutait plus volontiers les chansons populaires de Georges Wassouf et de Rami Ayach dans la voiture quand il allait se balader, ce sont bien les discours de Walid Joumblatt remixés ${ }^{31}$ qui passaient en boucle sur son ordinateur portable lorsqu'il révisait ses cours pour les examens à venir. Comme si la voix remixée de Walid bek

\footnotetext{
${ }^{27}$ L'un des frères a été enlevé et retenu prisonnier pendant plusieurs semaines pour faire pression sur l'autre, qui dirigeait l'établissement bancaire depuis l'Arabie saoudite.

${ }^{28}$ Elle sous-entend ici qu'elle pourrait avoir besoin du soutien financier de la famille de son mari; par conséquent, il ne serait pas bon d'afficher une relation avec Walid Joumblatt en lui rendant visite.

${ }^{29}$ "Celui qui éduque, le professeur, le maître ", surnom très couramment employé pour désigner Kamal Joumblatt. La production écrite de Kamal Joumblatt est assez conséquente et comprend des essais à vocation philosophique et ésotérique, de la poésie, des essais d'analyse politique. L'essentiel de cette production a été édité ou réédité par Dar al-Taqaddoumiyya), la maison d’édition fondée par Walid Joumblatt à Moukhtara.

${ }^{30}$ Rivoal, 2011 (à paraître).

${ }^{31}$ Notamment le plus populaire, celui qui a été prononcé sur la place des Martyrs, le 14 février 2006, à l'occasion de la commémoration de l'assassinat de Rafic Hariri, et qui s'attaque à Bachar al-Assad, le président syrien, le qualifiant de singe, de serpent et de requin. La scène décrite ici se déroule le mois suivant, en mars $\underline{2007}$.
} 
exhortant les "fils de la Montagne » au courage et à l'abnégation était le meilleur moyen de se motiver - ou peut-être de conjurer le sort.

Enfin, la familiarité passe aussi par une construction de la présence du leader dans un espace présenté comme " chez lui » et donc " chez nous ». "Lorsque Walid bey remonte dans le Chouf pour le week-end, il n'utilise jamais sa voiture avec une plaque de ministre ou de député. Tu vois, son $4 \times 4$ immatriculé 27000 , tout le monde le connaît ici ${ }^{32}$ ! » Ou encore : " Moi, je me souviens, quand Walid bey allait chercher Taymour [son fils] à Deir el-Qamar chez Patrick Perez [au Centre culturel français], il arrivait la veste sur l'épaule. " À la différence de ce qu'avait noté Michael Gilsenan, le "seigneur » ne se met pas en scène sur le mode d'une distance lointaine et surplombante par rapport à ses partisans. C'est au contraire l'image d'un homme décontracté et affranchi des codes politiques et vestimentaires de la ville que les gens aiment rendre quand ils évoquent ses déplacements dans la région. On peut y voir comme une extension métaphorique du dispositif inscrivant le leader politique dans l'intimité des maisons : dès qu'il entre dans le Chouf, Joumblatt est "chez lui ». Si l'on fait ici référence à la première des propriétés impliquées dans la constitution du home selon Ghassan Hage, soit la possibilité d'un espace où l'on ne subit plus la loi de l'autre, on pourra dire que la référence à un "chez-soi ", dans ce contexte, doit être comprise comme un espace social d'où le défi et la compétition politique ont été neutralisés, sinon évacués.

Le concept de "connectivité patriarcale" proposé par Suad Joseph dans ses travaux sur la construction des relations familiales au Liban éclaire de façon pertinente la nature ambivalente de cet espace hiérarchisés ${ }^{33}$. La connectivité désigne, selon elle, une propriété fondamentale de la construction de la personne dans les sociétés arabes qui suppose de se penser en relation aux autres référentiels ${ }^{34}$ et non pas comme sujet autonome. Autrement dit, la maturité d'une personne sera évaluée dans sa capacité à répondre aux désirs de ses proches - dans la sphère familiale et, par extension, l'ensemble des personnes qui sont susceptibles d'être considérées ${ }^{35}$-, à anticiper leurs besoins, à parler les uns pour les autres, à prendre en compte ce qu'ils aiment ou ce qu'ils n'aiment pas. Tout au long du cycle de vie, les personnes font l'expérience de modalités de connectivité différentes, se voyant comme des extensions des autres ou voyant les autres comme des extensions d'elles-mêmes. Les relations peuvent être fondées sur l'affection ou, au contraire, sur l'hostilité. Cette manière de se construire en relation aux autres est orientée dans un sens patriarcal : les hiérarchies sont avant tout des hiérarchies relationnelles. Les hommes sont élevés pour être en mesure de répondre à, et de demander l'investissement des autres dans leur vie. C'est en ce sens qu'ils dominent car ils ont l'initiative - initier la conversation, initier l'interaction... Mais ils ont besoin des autres pour se réaliser complètement ${ }^{36}$. Leurs actions dans ce contexte sont toujours enchâssées

\footnotetext{
32 Les ministres, députés et hauts fonctionnaires circulent en général en Mercedes dernier modèle. La plaque minéralogique spécifique à la fonction permet de les identifier.

33 Joseph, 1993 et 2005.

${ }^{34}$ Suad Joseph utilise le terme de significant others.

${ }^{35}$ Voir le concept de "proximité » (qarâba) proposé par Dale Eickelman comme extension pragmatique de la notion de parenté (Eickelman, 1981, p. 109).

${ }^{36}$ D'où l'importance de fonder un foyer : on n'est pas un homme complet si l'on n'a pas d'autres qui soient des extensions de soi-même. Plus on a d'extensions de soi, plus on est important. La charge de « représenter " son groupe en est l'expression la plus accomplie. Pour une analyse de tout ce qu'implique la compétence de " maître de maison », voir Jungen, 2011 (à paraître).
} 
dans une matrice relationnelle: on ne gagne en importance et en reconnaissance sociale qu'en relation à la subordination d'un grand nombre de personnes à même de répondre à ses attentes.

Ces références à Walid Joumblatt comme étant décontracté " chez lui », dans le Chouf, soulignent la construction d'une figure de maître de maison ${ }^{37}$. C'est en ce sens que l'on peut comprendre la place qui lui est donnée dans l'intimité des gens: le leader est inscrit dans le quotidien de ceux qui lui reconnaissent cette place et dans leur intimité en ce sens qu'il est une extension d'eux-mêmes et qu'ils se voient comme une extension de lui. II fait indubitablement partie de ces personnages de référence qui participent de la construction des matrices interactionnelles au niveau local. Et cette construction a des implications sur les attentes réciproques du leader et de ses partisans.

\section{2-L'humeur du bey, ou de l'art de la politique à la libanaise}

La " personnalité " de tout individu avec lequel on entre en relation est soumise à une élaboration narrative dans le groupe. Des anecdotes le concernant, ses goûts, ses manières d'être ou de faire seront régulièrement évoqués ou relatés dans le cercle familial et à l'occasion de visites de personnes extérieures à ce groupe. C'est de cette manière que l'on prend place dans une matrice relationnelle donnée ${ }^{38}$. Il n'est dès lors pas étonnant que les gens évoquent avec plaisir le caractère et la personnalité de Walid Joumblatt. II existe même une attente à ce qu'il se donne à voir d'une manière qui permette ces élaborations : le leader doit avoir " un style » qui le distingue des autres. Plus ce style sera établi, plus il sera la marque d'une accumulation biographique qui vaut légitimité ${ }^{39}$. Un leader qui a un style reconnu est aussi un leader qui offre une certaine matière susceptible d'élaborations. Au plus proche de la construction intime, les femmes savent quels sont les plats préférés de Walid bey. Les jeunes se régalent de visionner sur YouTube cette vidéo du " président » sirotant son maté, qu'il préfère amer. "Walid bey, il aime faire de la photo, il aime rouler à moto, il aime se promener seul dans le Chouf sans ses gardes du corps. D'ailleurs, il conduit toujours luimême sa voiture, et Marwan Hamade est à ses côtés. II n'aime pas lire comme son père, mais il est intelligent et rusé, etc. " Ces goûts, habitudes, traits de personnalité sont évoqués à l'intention de l'ethnologue qui vient séjourner dans le Chouf, comme une manière de l'initier à ce savoir partagé. Dans les conversations qui animent les soirées en famille (sahra), on se demandera plutôt ce que Walid bey penserait de ceci ou cela en fonction de ce que l'on sait de lui, de ses choix, de sa personnalité. Cette manière d'impliquer Walid Joumblatt dans la conversation est plus souvent le fait

\footnotetext{
${ }^{37}$ Cette dimension est bien perçue par les chrétiens qui ont fui la Montagne durant les affrontements de 1983 et qui disent ne pas se sentir chez eux dans cette région, même s'ils y ont reconstruit des maisons. Ils n'acceptent pas de devoir agir comme s'il fallait montrer de la déférence aux druzes en se pliant au code de convivialité, la mujâmala (de Clerck, 2009). En ce sens, il n’est pas étonnant que la scène de Walid bey «la veste sur l'épaule " se déroule précisément à Deir al-Qamar, ville de l'autre (chrétien), symbolique dans le Chouf. Sur l'articulation symbolique des différents lieux du Chouf, voir Rivoal, 2001.

${ }^{38}$ Par exemple, l'ethnologue qui développe une relation privilégiée avec un ensemble de personnes peut ainsi évaluer son intégration sur le terrain à "l'épaisseur » de l'élaboration discursive dont il fait l'objet et à sa capacité d'y répondre et de conforter cette élaboration.

39 Le fait de pouvoir être plus que l'élément d'un groupe donné est l'une des catégories de socialisation fondamentale selon Simmel : "Que l'individu ne soit pas par certains aspects un élément de la société, c'est au contraire la condition positive pour qu'il le soit par d'autres aspects de sa nature : sa manière d'être socialisé est déterminée ou codéterminée par sa manière de ne pas être socialisé » (Simmel, 1999, p. 71).
} 
des jeunes gens qui en font une sorte d'oncle maternel, associé affectueusement dans leurs choix de $v^{4} e^{40}$. Walid Joumblatt est une référence importante au quotidien. Mais cette référence n'est pas seulement une référence pour soi, elle est également un enjeu dans les relations entre les personnes.

L'art de la discussion politique au Liban consiste souvent à produire une analyse des intentions des différents acteurs dans une situation donnée. L'intention est souvent plus pertinente que l'acte ou le discours, plus sujette à conjecture. Elle offre la possibilité d'un " jeu » entre celui qui élabore une analyse et ceux à qui elle est destinée. Tout l'art du jeu consiste à produire à la fois des informations attestées, qui sont une manière de donner à voir ses relations, et à pointer les signes et les indices qui sont la sécrétion de l'intention ${ }^{41}$. Cette manière de faire est particulièrement bien illustrée dans l'échange que j'ai eu avec le responsable de la branche du PSP à Sydney. Le Dr Mamdouh disait qu'il lui fallait passer plusieurs heures, tous les jours, à lire les journaux libanais, syriens et saoudiens, m'expliquant qu'il faut apprendre à lire entre les lignes: " Par exemple, ce matin [1 ${ }^{\mathrm{er}}$ avril 2008], le Nahâr disait seulement dans un petit article que Walid bey avait été vu sur la route de l'aéroport avec la vitre de sa voiture baissée. Voilà ce à quoi il faut faire attention, parce que cela veut dire qu'il est détendu. Et moi je peux te dire qu'il est détendu parce qu'en ce moment, c'est le sommet arabe à Damas et il ne peut rien arriver à sa sécurité ${ }^{42}$. " Néanmoins, même pour celui qui sait " lire comme ça ", il en faut plus pour comprendre la politique quand on est loin : " C'est pourquoi j'appelle régulièrement les députés, Wa'el Abou Fa'our, Ghazi al-'Aridi. Ghazi surtout parce qu'il est de ma région. Mais eux, ils ne donnent pas vraiment les informations. Alors le meilleur moyen de rester informé, c'est d'appeler les anciens camarades (rifâq). Eux, ils sont sur place, ils parlent et ils disent ce qu'il se passe. Et bien sûr, j'appelle régulièrement à Moukhtara. Il y a toujours un jeune qui répond et qui donne des nouvelles. Comme ça, on sait si Walid bey est contrarié, s'il est obligé de faire quelque chose qu'il ne veut pas ou bien, au contraire, s'il est de bonne humeur. Là, on peut garder le contact. »

Les séances d'audience que Walid Joumblatt donne tous les samedis matin dans sa résidence de Moukhtara sont le lieu particulier où est mise en scène la dimension personnelle caractérisant la relation de patronage. C'est là bien sûr que l'on vient demander une recommandation pour une embauche, une aide financière en cas d'hospitalisation, ou que l'on vient exposer un problème, demander une médiation, apporter une invitation. Dans cet espace politique par excellence du diwân oriental, le leader agit comme un chef d'orchestre autant qu'il reçoit. La scène n'est jamais figée dans une situation d'audience. C'est sur une chaise en plastique tournant le dos au balcon que Walid Joumblatt s'installe, ses visiteurs se retrouvant disposés sur les canapés qui courent le long des hauts murs de son salon. Selon les visiteurs qui prennent place à tour de rôle, il discute à voix basse ou au contraire manifeste son agacement à voix haute devant la teneur de la requête (" vous me faites perdre mon temps et mon café avec votre histoire ! »). Ou encore, il se lève soudain, montre de la compassion pour l'un d'eux, à qui il donnera directement quelques billets sortis de son portefeuille

\footnotetext{
${ }^{40}$ Rivoal, 2011 (à paraître).

${ }^{41} \mathrm{~J}$ 'emprunte à Andras Zempléni la notion de sécrétion comme processus organisé de fuites autour d'un secret (Zempléni, 1976).

${ }^{42}$ La scène a lieu un mois avant les affrontements de mai 2008 qui se sont déroulés à Beyrouth et dans le Chouf entre le Hezbollah et les partisans de Hariri et de Joumblatt. La tension était alors à son plus haut point et, implicitement, mon interlocuteur pointait le danger qu'il y avait à traverser la banlieue sud de Beyrouth.
} 
personnel, puis prend l'invitation tendue tout en regardant déjà ailleurs (" Bon, qui est le suivant ? Suivant!»), retourne s'asseoir subitement perdu dans ses pensées avant de se relever pour accepter, avec un sourire, l'embrassade sur le front d'une vieille qui lui souhaite longue vie; l'instant d'après il s'exclame qu'il en a assez qu'on allonge constamment sa vie, qu'il voudrait qu'on le laisse tranquille ${ }^{43}$. Joumblatt semble changer constamment de registre, acceptant les marques de déférence pour tancer ensuite celui qui avance devant lui une table à café afin qu'il y dépose son agenda (« Ces tables sont faites pour le café, enfin ! »). II se lève, s'assoit, passe sur le balcon, discute en aparté avec ses " administrateurs ${ }^{44}$, puis il disparaît dans une autre pièce pour revenir quelque temps après, faisant ainsi attendre une délégation venue lui rendre visite.

Le diwân est plus ici que ce lieu de la parole et de l'affirmation des hiérarchies statutaires que la littérature a décrit. II est aussi la mise en scène d'une indéniable tension entre le leader et ceux qui lui rendent visite, une tension qui s'exprime comme "l'humeur du bey». Ce dernier en joue notamment en changeant constamment le "rythme » de ces séances d'audiences, comme s'il s'attachait précisément à rester imprévisible devant un public pour lequel une part de l'habileté politique consiste à trouver les moyens de lire son action et à le rendre prévisible. Mais cela aussi fait partie du "style » de Walid Joumblatt. Le bey ne s'installe pas hiératique dans son salon pour dire « regardez-moi ». Ce qu'il donne à voir est ailleurs, dans la disposition des portraits familiaux qui ornent les murs des différents salons qui sont ouverts au public, dans le jardin raffiné qui s'étend sur les terrasses du palais avec ses gazelles, ses fleurs odorantes et ses cascades d'eau qu'il permet à des visiteurs choisis de parcourir " en touriste ", dans la bibliothèque d'une aile attenante qu'il fait restaurer avec soin et qui le met en scène en compagnie de Kamal Joumblatt sur la scène politique internationale. Dans son salon, les jours de réception, Walid Joumblatt n'est pas cette image de luimême avec laquelle les gens jouent chez eux, il agit, il met en scène tous les registres de ce que signifie être un seigneur de la Montagne. II doit déployer une action qui révèle les multiples facettes de la relation que ses clients ont avec lui. II n'est pas une référence iconique qui se donnerait d'un bloc, prévisible et immuable. II doit répondre aux attentes, sans jamais se laisser enfermer dans la relation, même si les gens sont aussi conscients qu'il existe localement par cette mise en scène hebdomadaire, comme le révèle ce commentaire d'un critique de Joumblatt : " $\mathrm{Si}$ un jour personne ne montait à Moukhtara, crois-moi, le bey se suiciderait... »

La proximité politique avec le leader ne s'établit donc pas uniquement dans les termes d'une relation de clientélisme, qu'on y voie un effet de la tradition ou l'inscription dans un type d'échange. La dimension personnelle de la relation patron-client ou leader-partisan suppose une aptitude à lire I'homme politique dans ses attitudes et ses humeurs, non pas parce que cette lecture aurait des effets sur soi-même, comme on jauge l'humeur du chef de service en arrivant au bureau le matin, mais parce qu'il s'agit d'une information essentielle dans le décodage politique, même quand on a émigré, et parce que cette participation est une manière d'appartenir. Être en mesure de parler de l'humeur du bey, c'est montrer qu'on fait partie du cercle de proximité. II s'agit d'une compétence qui organise une certaine forme de compétition entre partisans. La proximité avec le leader politique

\footnotetext{
${ }^{43}$ En référence à la formule constamment réitérée "Alla(â)h itawwil 'omrak('umrak) » (Que Dieu allonge ta vie), Joumblatt a répondu(déclaré) : "hellu(û?) 'anni(î) wa ma ttawulu(û) wa ma tkasru(û) 'omri('umrî)! " (Laissez-moi tranquille et n'allongez pas ni ne raccourcissez ma vie !).

${ }^{44}$ Qui est chargé de considérer les problèmes de permis de construire, qui d'embauches sur les quotas de la police ou de l'armée, qui des affaires médicales...
} 
n'est pas ici l'apanage d'un groupement identifié d'hommes de main dont le statut social serait directement fondé sur cette relation privilégiée. Le leader définit un espace de pertinence politique qui peut se lire en termes de distance constamment négociée avec la référence qu'il représente. Une référence qui est double, car Walid Joumblatt ne saurait être sans la référence à Kamal bey, son père martyr.

\section{3- La pérennité d’un « pôle " politique de la Montagne}

Il reste à questionner ce que construit la relation entre un leader et ses partisans dans la forme spécifique que nous avons décrite. Cette forme rejoint l'évaluation proposée par Gérard Lenclud dans son analyse des relations de patronage en Corse, qui s'était interrogé sur le fait qu'elles étaient inscrites de façon hautement visible dans l'orbite de l'affection et de l'amitié. La caractéristique de toute relation est de créer un certain nombre d'attentes. Ces attentes, que l'on peut aussi conceptualiser comme l'expression d'un désir, en sont une dimension particulièrement valorisée au Liban, comme l'a bien analysé Suad Joseph ${ }^{45}$. Associées à la négociation permanente d'un quant-àsoi autorisant la compétition, ces attentes fondent l'ordre politique, et leur ritualisation construit les sociétés partisanes. Car s'il existe une éthique de la relation et une étiquette dans la familiarité avec le seigneur, ce sont les formes ritualisées de l'échange qui construisent les espaces politiques régionaux au Liban. Ces "pôles" ne concentrent pas seulement un pouvoir de nature communautaire, ni n'exercent une domination contre la société, ils condensent un surplus de vie et un sens du possible. La ritualisation des visites permet de saisir pleinement cette dimension. La " polarité " est bien sûr construite dans ces visites que les partisans rendent tous les week-ends à Moukhtara. II n'est pas besoin d'avoir une demande à formuler, un projet à exposer, on peut " monter chez le bek» simplement pour voir des amis, prendre des nouvelles, être au palais. Ces audiences permettent d'inscrire Walid Joumblatt dans le rythme hebdomadaire, d'organiser le temps à l'échelle d'un espace dont les confins sont définis par les villages d'où plus personne ne se rend régulièrement à Moukhtara. L'espace politique de la relation n'est pas forcément celui du territoire marqué par les check-points de la guerre civile.

En retour, Walid Joumblatt et, depuis peu, son fils Taymour, rendent visite chaque mois aux familles qui ont connu un deuil. La formule rituelle lors d'une visite de condoléances veut que l'on souhaite à chacun des proches du défunt le surplus de vie laissé par celui-ci : " al-'awad bi-salâmtak " ${ }^{46}$. Or, lorsque le seigneur de Moukhtara offre ses condoléances, c'est à lui que les parents du défunt retournent la formule. Ce surcroît de vie souhaité au seigneur, on l'a vu, est une dimension importante de l'échange direct avec le leader, à tel point qu'il arrive à Walid Joumblatt de manifester son agacement, comme s'il voulait marquer par là qu'il ne saurait être redevable du don qui lui est ainsi fait. Dans le contexte de funérailles, en revanche, il inscrit le seigneur dans le cercle des proches du défunt. Arrivé en visiteur, il est transformé en représentant de la maison qu'il visite. Le rituel actualise ici la mise en scène plaçant le leader politique au centre de la vie familiale. On observe une

\footnotetext{
45 Joseph, 2005.

${ }^{46}$ Elle repose sur l'étalon qu'une vie humaine est prévue pour durer cent ans. La formule employée pour souhaiter l'anniversaire à quelqu'un(souhaiter à quelqu'un son anniversaire) est ainsi « que tu atteignes cent ans ". Quiconque décède avant cet âge canonique laisse donc un « surplus " d'années dont on souhaite à ses proches de profiter.
} 
transformation similaire dans la manière dont les religieux acceptent la poignée de main de Walid Joumblatt quand la règle veut en effet qu'ils mettent la main sur le cœur pour saluer tout homme qui $\mathrm{n}^{\prime}$ appartient pas au cercle de leurs proches ${ }^{47}$. Walid Joumblatt formalise cette interdépendance en tenant sa propre famille à distance. Les seuls portraits qu'il expose dans son salon sont ceux de son père Kamal, de sa grand-mère Sitt Nazira, et des chauffeurs morts dans les attentats l'ayant visé et ayant tué son père. En somme, le témoignage de la lignée politique et des martyrs qui en construisent la légitimité politique. La référence à sa propre famille, ou ce qui passerait pour le clan Joumblatt, est totalement effacée de l'espace public et des discours à tel point qu'il est quasi impossible d'obtenir des informations sur la parenté de Walid bey en questionnant les habitants du Chouf, d'ordinaire si diserts sur les seigneurs de Moukhtara. Ils semblent ne pas savoir. Et c'est cet oubli qui permet au seigneur d'occuper cette place particulière dans les familles qui forment sa clientèle politique.

C'est cependant dans le traitement des martyrs que se donne le mieux à voir la captation du surcroît de vie laissé par la mort. La construction du martyre est centrale dans toutes les communautés libanaises, même si les modalités et les implications sont différentes. Être martyr, c'est avoir un destin libanais, selon la formule d'Antoine Messara ${ }^{48}$. En ce sens, le martyre pourrait à l'instar de la communauté être considéré comme lieu du politique, qui ne pourrait se saisir pleinement que dans l'étude comparée de ses différences régionales. Avec le martyr, c'est un travail social particulier sur la violence qui est réalisé, une manière de la sortir des logiques de confrontation pour fonder ou refonder. Dans la communauté druze, les martyrs ne sont pas pris en charge par l'extérieur à titre individuel, comme cela peut être le cas dans la communauté chiite ou pour les sunnites du Akkar ${ }^{49}$. Ils sont tous fondus dans la figure paradigmatique de Kamal Joumblatt, le martyr par excellence. Le Chouf, dont on a vu qu'il était construit comme l'espace domestique de Walid Joumblatt, est très pauvre en calicots et portraits du seigneur. En cela, il contraste avec la plupart des régions libanaises. La principale sinon la seule présence politique passe par l'évocation de Kamal Joumblatt: généralement des plaques ou de petits cénotaphes en son honneur sur les places des villages. Et c'est toutes les routes de la région qui s'illuminent des petites bougies déposées par les Jeunesses du PSP le 6 décembre, sa date de naissance. Le martyre de Kamal articule moins aujourd'hui une rhétorique guerrière qu'il ne permet une lecture sacrificielle par la construction du leader assassiné en 1977 comme figure quasi christique. " Kamal bey comme d'autres Joumblatt avant lui ont versé leur sang sur la terre du Chouf, c'est grâce à ce sacrifice que les gens de ce pays sont encore là. »

Il semble que la mort violente soit traitée comme si elle devait être absorbée dans la figure de Kamal Joumblatt. Lorsque deux jeunes de Baaqline, membres des Jeunesses du PSP, sont décédés dans un accident de voiture, l'hommage que leurs camarades ont imaginé a été d'aménager un lieu à leur mémoire dans lequel on vendrait les livres de Kamal Joumblatt. Cette manière de traiter les morts en les associant à une autre figure pour assurer la pérennité de leur mémoire personnelle évoque l'enterrement dans la proximité du tombeau d'un saint. C'est peut-être ainsi que l'on doit considérer le monument grandeur nature consacré aux martyrs de la guerre civile, associés dans cette représentation aux combattants de 1860, à Duwwar (entre Baaqline, Beiteddin et Baq'ata), érigé au

\footnotetext{
${ }^{47}$ Il existe par ailleurs une manière spécifique de se saluer entre religieux.

${ }^{48}$ Messara, 1988.

${ }^{49}$ Sur l'implication du Hezbollah au sud, du parti Mustaqbal et de l'armée au nord, pour les martyrs des affrontements à Nahr el(al)-Bared, voir Volk, 2009.
} 
centre d'un territoire que balise la mémoire de Kamal Joumblatt ${ }^{50}$. La dimension religieuse à travers laquelle la figure du martyr est réélaborée permet à Walid Joumblatt de construire sa propre clôture tout en demeurant une référence active car le pôle de Moukhtara s'appuie sur une lignée et continue d'écrire l'histoire de la région. II ne s'agit pas seulement d'une lignée qui capterait I'histoire par sa domination. Le leadership Joumblatt ne peut se saisir uniquement dans la personne de Walid bey, comme il n'existait pas complètement dans la figure de Kamal avant lui, qui devait revendiquer également d'autres morts violents dans la lignée (son propre père, mais surtout la référence paradigmatique au cheikh Bachir Joumblatt, éliminé par l'émir Bachir Chihab au XVIII ${ }^{\mathrm{e}}$ siècle). L'image du leader est ici dédoublée et le martyr est plus qu'une référence mémorielle. Ainsi quand Ibtissam fait son yoga, elle rend Kamal présent: il faut avoir une relation au mort pour s'assurer une vie meilleure, fût-ce par le biais de la méditation. C'est ce qui donne à la lignée politique une profondeur historique et une légitimité en tant que leadership de la Montagne.

\section{4- De la bonne distance}

Mais cette légitimité fonctionne comme une polarité, un espace d'influence, de négociation, d'attentes et non comme une autorité instituée. C'est bien ce qu'il faut comprendre pour saisir la particularité des enjeux politiques et sociaux au Liban : rien ne se donne en dehors de la relation au leader (constitution de la famille, construction de l'espace, situations quotidiennes saturées de références), mais cette relation n'est jamais fixe. Les chefs de familles, les femmes en cuisine, les jeunes, chacun exprime une facette différente de la relation au leader, on l'a vu. La morphologie n'épuise pas le sens de cette relation. Comprendre ce qui fait du Liban ce "théâtre des passions politiques " analysé par Raymond Jamous ${ }^{51}$, c'est aussi observer l'intensité de la relation, comment on en joue et comment chacun considère et commente le jeu des autres. Le politique au Liban, $c^{\prime}$ est une tension mise en spectacle à laquelle la seule alternative est le « hors-jeu ».

C'est autour d'une métaphore de la distance qu'est évoquée la domination politique : « Walid bey, tu vois, il estcomme le soleil : quand on est trop loin, on gèle, mais quand on est trop près, on brûle, il faut savoir trouver la bonne distance. ". Souha m'avait fait cette réflexion alors que je lui avais rendu visite sur le chantier de la maison qu'elle bâtissait dans le Metn pour un banquier druze. J'avais fait sa connaissance dans le cadre d'une enquête sur le conseil municipal de Baaqline dont elle était membre et avais eu l'occasion de lui rendre visite à plusieurs reprises. Proche de Walid Joumblatt, elle l'était certainement, mais elle s'était fâchée avec lui pour des problèmes de construction et désormais, me disait-elle alors, elle évitait d'aller dans les réunions auxquelles il participait. Le mois suivant, j'étais) surprise de retrouver Souha en visite à Moukhtara. Après une brève discussion avec Walid Joumblatt dont je n'avais pu saisir la teneur dans le brouhaha ambiant, elle était allée converser avec le responsable des travaux publics du " palais " (balât), me saluant tout de même au passage d'un très léger signe de la tête. L'après-midi avait lieu à Baaqline une exposition des réalisations de l'artisanat du Chouf dans la Maison communale (bayt Ba'aqlin). Avec étonnement, je

\footnotetext{
${ }^{50}$ Il faut toutefois signaler un autre monument aux martyrs de la guerre sur la place centrale de Dayr(Deir) alQamar, qui est polarisé par Dany Chamoun, fils du leader chrétien Camille Chamoun qui fut le grand adversaire politique de Kamal Joumblatt dans la région.

51 Jamous, 2004.
} 
vois Souha me croiser en évitant ostensiblement de me reconnaître cette fois, ne s'arrêtant pas, même brièvement, pour échanger quelques mots sur notre présence le matin même dans le diwân de Walid bey.

La réitération, dans des contextes différents, de cette forme d'évitement m'a amenée à la considérer comme une attitude sociale pertinente au Liban, qui n'engage pas la relation sur le fond. Ainsi, quelques années plus tard, j'ai été amenée à recroiser Souha lors d'une manifestation publique et elle $\mathrm{m}$ 'a alors chaleureusement saluée. Je suggère de comprendre ce genre d'attitude alternant évitement et reconnaissance de l'autre comme une manière de négocier des relations dans un rapport à un tiers significatif, qu'il s'agisse d'une personne particulière ou d'un public. Dans cette scène, l'ethnologue qui avait fonction à rester à la marge du jeu politique régional se retrouvait inopinément dans un espace dont l'une des fonctions est l'exposition des intentions et des projets en cours de chacun. Dans son travail sur le secret, Simmel a souligné que la nécessité sociologique du savoir réciproque des hommes les uns sur les autres (qu'il s'agisse de préjugés ou d'informations communiquées dans l'interaction) implique également que " tout être humain est entouré d'une sphère invisible dont la dimension peut varier selon les différentes directions et les différentes personnes auxquelles on s'adresse " $^{52}$. C'est la distance du quant-à-soi devant les autres qui rend manifeste la distance nécessaire entre les personnes. Le fait de distinguer chaleureusement quelqu'un dans un groupe, de prendre à part, de notifier très discrètement ou encore d'ignorer ostensiblement, tout cela participe de la manifestation d'un espace entre ce que l'on est et ce que I'on donne à voir (ou que l'autre ne devrait pas voir) ${ }^{53}$. Il semble que l'on soit ici dans un contexte où le quant-à-soi devant les autres se construit en partie dans l'actualisation permanente de la relation au leader : trop près, et comme dans l'analyse de Simmel on anéantit la sphère de ce qui nous est personnel (le valet); trop loin, et l'on perd la possibilité de la " distinction " conférée par le leader dans le jeu politique consistant à montrer que l'on est assez proche pour connaître son humeur. Si la politique au Liban peut être décrite comme relevant du registre de la "passion " c'est en raison de l'intensité de l'activité interprétative qu'elle suppose.

Cette attitude qui est la plus courante n'est cependant pas la seule manière de se positionner en relation à Walid Joumblatt. Une autre manière de se définir consiste à refuser la relation et le jeu de déchiffrage constant, de rapprochement/éloignement qui va avec, en tentant d'enfermer le leader dans un rôle de référent omnipotent hors d'atteinte. C'est la position que traduit Nassim, un ancien acteur de la guerre civile. Peu de temps après avoir accepté de m'héberger pour les besoins de mon enquête, et alors que je commençais à vouloir rencontrer des personnes dans son village, il me mit en garde : "Walid bey est la porte du Chouf, il sait tout sur tout le monde et s'il ne veut plus de toi ici, tu ne trouveras personne, pas même un hôtel, pour t’héberger ! » II me rappelait constamment qu'ici j'étais sous sa protection et que Charif Fayyad, le secrétaire général du PSP, l'appelait tous les jours pour savoir qui je voyais et ce que je faisais. Cette construction d'un leader omnipotent et omniscient allait de pair avec l'absence de ces signes de la présence du leader dans la vie quotidienne que j'ai observés à maintes reprises ailleurs - mis à part le portrait de Kamal Joumblatt dans le salon, aucune marque "d'intimité " n'était rendue manifeste. Jamais non plus, je n'ai vu

\footnotetext{
${ }^{52}$ Simmel, 1999, p. 358.

53 Dans le cadre de cette analyse sur la distance et le jeu sur savoir, montrer, taire et mentir, voir Gilsenan, 1996, et Jamous, 1993.
} 
Nassim monter à Moukhtara durant le week-end. Blessé lors de l'attaque de son village par les Forces libanaises en 1983, il montrait sa blessure au bras en se présentant comme un homme fort, celui que l'on vient voir quand il y a un problème dans le village, qui sait parler aux jeunes et s'imposer. Mais en réalité, Nassim était souvent seul sur sa terrasse et ne recevait guère de visites en dehors de ses frères et de ses oncles. Au-delà de la fanfaronnade, il faut noter comment ce personnage construit, en contraste avec la majorité des gens, une distance maximale avec le leader politique. Au plus proche de la configuration analysée par Michael Gilsenan, la ressource pour éloigner le seigneur consiste à jouer sur "l'homme de main ", relais et médiateur. Le secrétaire du parti est présenté comme la seule relation effective avec le pouvoir, encore qu'elle soit uniquement mentionnée comme relation téléphonique, ce qui n'est pas sans évoquer les modes de communication de la période de guerre. Tout en reconnaissant la domination sans partage de Joumblatt, il refuse la relation concrète avec l'homme politique et ne dit rien de lui ; cette "transcendance " assignée se manifeste par l'impossibilité de lui faire une place dans le quotidien. Cette manière de refuser la relation avec le seigneur est une façon de se construire soi-même au centre d'un espace de domination - dans le cas de Nassim, son gynécée essentiellement. Comme si les stigmates de la violence qu'il arbore l'autorisaient à se poser comme duplication d'un idéal-type incarné par Walid Joumblatt, alors qu'il apparaît en fin de compte comme un personnage tragique dont la mise en scène "tourne à vide " parce qu'il n'a pas été en mesure de sortir d'une certaine logique sociale, celle de la guerre civile ${ }^{54}$.

\section{Conclusion}

La perspective anthropologique que j'ai développée dans cet article est adossée à l'idée que le Liban ne peut être compris qu'à partir de totalités partielles se définissant réciproquement dans un jeu politique d'alliance et de confrontation; des expressions politiques localisées qui ne sont jamais closes sur elles-mêmes et qu'il faut décrire dans leur complexité. On ne peut épuiser la compréhension du politique au Liban en décrivant le pouvoir tel qu'il se manifeste dans le système confessionnel et les relations de patronage sous le seul rapport du contrôle des ressources et de l'asymétrie. La relation patron-client ne se donne pas comme une dualité dans laquelle les termes se définiraient de manière réciproque et univoque. L'espace de la relation est beaucoup plus complexe et suppose de construire l'ensemble des manières dont elle se déploie au quotidien : formes de l'intimité entre le leader et ses partisans, acceptation et refus par le leader de cette place assignée qu'il manifeste par ses rebuffades publiques, construction d'une dualité nécessaire dans la figure politique. Cette asymétrie fonctionne plutôt à la manière d'une polarisation posant la question de la zone d'influence et du fonctionnement de cette influence. Autrement dit, il y a beaucoup plus que du pouvoir dans la relation de patronage : de multiples registres de définition "en relation à " qui ne peuvent jamais être présupposés parce qu'ils dépendent des contextes locaux et qu'ils sont constamment modifiés par le jeu lui-même.

\footnotetext{
${ }^{54}$ Rivoal, 2009.
} 


\section{Bibliographie}

Beyhum Nabil, 1999, "Beyrouth, histoire de deux villes où tuer est une compulsion qui se répète ", dans Hannoyer J. (dir.), Guerres civiles. Économies de la violence, dimension de la civilité, ParisBeyrouth, Karthala-CERMOC, p. 123-138.

Clerck Dima de, 2009, "Guerre, frontière et rupture identitaire dans le sud du Mont-Liban. Les relations revisitées entre druzes et chrétiens de la Montagne", Vingtième siècle. Revue d'histoire, $\mathrm{n}^{\circ} 103$, p. 163-176.

Dib Kamal, 2004, Warlords and Merchants. The Lebanese Business and Political Establishment, Reading, Ithace Press.

Donegani Jean-Marie et Sadoun Marc, 2007, Qu'est-ce que le politique ?, Paris, Gallimard.

Eickelman Dale, 1981, The Middle East. An Anthropological Approach, Londres, Prentice Hall.

El-Ghoussayni Noha, 1988, Émergence d'une nouvelle organisation régionale au Liban: le Chouf, thèse de doctorat, Institut d'urbanisme et d'aménagement de la Sorbonne-Paris IV.

Freund Julien, 1965, L'Essence du politique, Paris, Sirey.

Gubster Peter, 1973, "The Zu'amâ of Zahlah: The Current Situation in a Lebanese Town ", The Middle East Journal, 27(2), p.173-189.

Gilsenan Michael, 1977, "Against Patron-Client Relations », dans Gellner E. et Waterbury J. (eds), Patrons and Clients in Mediterranean Societies, Londres, Duckworth, p. 167-183.

---- 1986, «Domination as a Social Practice. Patrimonialism in North Lebanon: Arbitrary Power, Desecration and the Aesthetics of Violence ", Critique of Anthropology, 6(1), p. 17-37.

---- 1989, "Words of Honour ", dans Grillo R. (ed.), Social Anthropology and the Politics of Language, Londres-New York, Routledge, p. 193-221.

---- 1996, Lords of the Lebanese Marches. Violence and Narrative in an Arab society, Londres-New York, I.B. Tauris.

Hage Ghassan, 1997, "At Home in the Entrails of the West. Multiculturalism, "Ethnic Food" and Migrant Home-building ", dans Grace H., Hage Gh., Johnson L., Langsworth J. et Symonds M., Home/World: Community, Identity and Marginality in Sydney's West, Annandale, Pluto Press, p. 99-153.

Harik Judith P., 1993, "Change and Continuity Among Lebanese Druze Community: The Civil Administration of the Mountain, 1983-90 », Middle Eastern Studies, 29(3), p. 377-398.

Hichi Selim Hassan, 1973-1974, La famille des Djoumblatt du VII siècle à nos jours, Beyrouth.

Hudson Michael, 1968, The Precarious Republic. Political modernization in Lebanon, New York, Random House.

Jamous Raymond, 1993, "Mensonge, violence et silence dans le monde méditerranéen ", Terrain, $\mathrm{n}^{\circ} 21$, p. $97-110$.

---- 2004, « Le Théâtre des passions politiques », Terrain, n 43, p. 141-156.

Johnson Michael, 1986, Class and Clients in Beirut. The Sunni Muslim Community and the Lebanese State, 1840-1985, Londres and Atlantic Highlands, Ithaca Press. 
---- 2001, All Honorable Men. The Social Origin of War in Lebanon, Londres-New York, I.B. TaurisCenter for Lebanese Studies.

Joseph Suad, 1993, "Connectivity and Patriarchy Among Urban Working-Class Arab Families in Lebanon ", Ethos, 21(4), p. 452-484.

---- 2005, "Learning Desire. Relational Pedagogies and the Desiring Female Subject in Lebanon ", Journal of Middle East Women's Studies, 1(1), p. 79-109.

Jungen Christine, "Devenir un maître de maison dans le Sud jordanien", dans Rivoal I. (dir.), La catégorie des "jeunes" dans le monde méditerranéen, Ateliers du LESC, 2011 (à paraître).

Kanafani-Zahar Aïda, 2004, "La réconciliation des druzes et des chrétiens du Mont-Liban ou le retour à un code coutumier ", Critique Internationale, $n^{\circ} 23$, avril, p. 55-75.

Khalaf Samir, 2001, Cultural Resistance. Global and Local Encounters in the Middle East, Londres, Saqi books.

Khuri Fuad, 2004, Being a Druze, Londres, The Druze Heritage Foundation.

Kingston Paul, 2001, "Patrons, Clients and Civil Society: A Case Study of Environmental Politics in Postwar Lebanon ", Arab Studies Quarterly, 23(1), p. 55-72.

Lenclud Gérard, 1993, "S'attacher. Le régime traditionnel de protection en Corse ", Terrain, n²1, Liens de pouvoir, p. 81-96.

Messara Antoine, 1988, "The Challenge of coexistence ", "Prospects for Lebanon" Paper, Center for Lebanese Studies, octobre.

Picard Élizabeth, 1994, "Les habits neufs du communautarisme libanais », Culture et conflits, $\mathrm{n}^{\circ} 15$ 16, p. $49-70$.

---- 1997, "Le communautarisme politique et la question de la démocratie au Liban ", Revue internationale de politique comparée, vol. 4, n 3, p. 639-656.

---- 2001, "Une sociologie historique du za'îm libanais ", dans Chartouni Ch. (dir.), Histoire, sociétés et pouvoir aux Proche et Moyen Orients (travaux dédiés à Toufic Touma), tome 1, Paris, Geuthner, p. 157-172.

Rivoal Isabelle, 2001, " Baaqline, de l'Administration civile de la Montagne à la renaissance municipale. Réflexion sur le pouvoir local au Liban ", dans Favier A. (dir.), Pouvoirs locaux et municipalités au Liban, Les Cahiers du CERMOC, $n^{\circ} 24$, Beyrouth, p. 319-338.

---- 2009, "Un huit-clos(huis clos) ethnographique ou l'impossible enquête chez un ancien milicien libanais ", dans Fogel Fr. et Rivoal I. (dir.), La relation ethnographique, terrains et textes. Mélanges offerts à Raymond Jamous, Ateliers du LESC, $n^{\circ} 33$, mars, (http://ateliers.revues.org/document8217.html).

---- 2011 (à paraître), " “Vouloir de la politique”. Exaltation, parodie et proximité dans l'engagement politique des jeunes au Liban ", dans Rivoal I. (dir.), La catégorie des "jeunes" dans le monde méditerranéen, Ateliers du LESC.

Simmel Georg, 1999, Sociologie. Étude sur les formes de la socialisation, Paris, PUF.

Volk Lucia, 2009, "Martyrs at the Margins: The Politics of Neglect in Lebanon's Borderlands ", Middle Eastern Studies, 45(2), p. 263-282.

Zempléni Andras, 1976, "La chaîne du secret ", Nouvelle Revue de Psychanalyse, n 14, (Du secret), p. 315-324. 